\title{
High Angular Resolution Studies of Disks-the Millimetre
}

\author{
David J. Wilner \\ Harvard-Smithsonian Center for Astrophysics, 60 Garden St., \\ Cambridge, $M A 02138$
}

\begin{abstract}
I briefly review progress in high angular resolution observations of disks at millimetre wavelengths, with a focus on dust continuum observations of the disks surrounding low mass pre-main-sequence stars. I summarize the utility of observations at millimetre wavelengths, where dust emission is largely optically thin, and I touch on several recent directions, including imaging surveys using interferometers, testing physical models of disks with resolved observations, and the complexities introduced by stellar multiplicity.
\end{abstract}

\section{Introduction: Disks around Young Stars}

Considerable effort is now devoted to characterizing the properties of disks around young stars to extract information on the physics of planet building and mass accretion. These disks are naturally amenable to observations over a wide range of wavelengths because they have substantial radial and vertical gradients in the key physical parameters of temperature and density. This short review concentrates on high angular resolution observations of disks at millimetre wavelengths, in particular interferometric observations of thermal continuum emission from small particles in the disks surrounding low mass pre-main-sequence stars. These disk systems are thought to be close analogs of the early solar nebula, and the surrounding protostellar envelopes are no longer present. Other papers in these proceedings treat related topics, including technical aspects of millimetre observations (A. Sargent), disks at earlier evolutionary stages (M. Hogerheijde), disks around stars of higher mass (A. Gibb), and molecular line observations and disk chemistry (A. Dutrey).

\subsection{Why Observe Disks at Millimetre Wavelengths?}

Most of the disk material surrounding low mass stars beyond a few stellar radii is at low temperatures, well below $1000 \mathrm{~K}$, where dust provides the dominant source of continuous opacity. A large fraction of the disk energy is radiated in the far-infrared, a spectral range difficult to access from the ground and lacking large apertures in space. Early analyses of the spectral energy distributions of $\mathrm{T}$ Tauri stars provided strong support for disk geometries and indicated minimum outer radii of 10's to 100's of AU (Adams, Lada \& Shu 1987). Importantly, the long wavelength tail of the dust emission becomes readily accessible through atmospheric windows at submillimeter and millimeter wavelengths. In this regime, the dust emission is (almost) entirely optically thin, and therefore 
the observed emission is directly proportional to mass, weighted by temperature. The disk surface density structure may be probed directly only at these wavelengths where the emission is optically thin; for the canonical value of mass opacity $\kappa_{1.3 \mathrm{~mm}} \approx 0.01 \mathrm{~cm}^{2} \mathrm{~g}^{-1}$, disks remain transparent up to column densities of $\approx 100 \mathrm{~g} \mathrm{~cm}^{2}$, an amount typically exceeded only interior to disk radii of a few AU. In addition, emission from circumstellar dust dominates emission from the stellar photosphere, generally by orders of magnitude at millimetre wavelengths, and there is no contrast problem between the disk and star.

The detection of millimeter emission from a large fraction of nearby $\mathrm{T}$ Tauri stars using sensitive bolometers on large telescopes showed that disk masses are typically sufficient for the formation of planetary systems (Beckwith et al. 1990). The uncertainties in the mass estimates are dominated by the lack of detailed knowledge of dust properties, such as size, shape, and composition, which are subject to evolution within the dense disk environment. However, the wavelength dependence of the dust emissivity manifested at millimeter wavelengths provides clues to such changes, in particular the growth from sub-micron interstellar sizes that represents an essential step in forming planets (Beckwith \& Sargent 1991).

\subsection{What is "High" Resolution at Millimetre Wavelengths?}

While the detection of dust emission from disks at millimeter wavelengths is not difficult with modern instrumentation, the angular sizes of the disks are small, and spatially resolved observations remain challenging to obtain and interpret. Improved modeling of spatially unresolved data continues to result in progress (e.g. Chiang et al. 2001), but direct imaging observations of disks at many wavelengths is essential to form a complete picture, to verify the theoretical constructs, and to break model degeneracies.

For the large sample of $\mathrm{T}$ Tauri stars in nearby dark clouds like Taurus, Ophiucus and Chameleon at $\sim 140 \mathrm{pc}$, the $\sim 100 \mathrm{AU}$ diameter of our Kuiper Belt would subtend only $0 ! 7$. The technique of interferometry allows for millimetre observations with high resolution, typically $\sim 0$ "' 5 , where attention must be paid to the role of atmospheric phase fluctuations (radio "seeing"). The large number of operating millimetre interferometers include the venerable Very Large Array upgraded to $7 \mathrm{~mm}$ operation, the Berkeley-Illinois-Maryland-Association array, the Owens Valley Radio Observatory Millimeter Array, the IRAM Plateau de Bure Interferometer, and the Nobeyama Millimeter Array.

New facilities include the upgraded Australia Telescope Compact Array, which provides access at $3 \mathrm{~mm}$ to targets deep in the southern sky for the first time, and the Submillimeter Array on Mauna Kea, which opens the $0.85 \mathrm{~mm}$ and $0.45 \mathrm{~mm}$ atmospheric windows to observations with subarcsecond resolution. The highest resolution now available may be obtained with the Very Large Array augmented by the link to the Pie Town VLBA antenna, which reaches $0 ! 02$ at $7 \mathrm{~mm}$ with sufficient sensitivity to detect the innermost regions of $\mathrm{T}$ Tauri disks (Wilner 2002) These new facilities will pave the way for the Atacama Large Millimeter Array, now at the start of a decade of construction work in northern Chile, which will provide revolutionary sensitivity, resolution and image quality for disk studies at millimetre and submillimetre wavelengths (see http://www.eso.org/projects/alma/). 

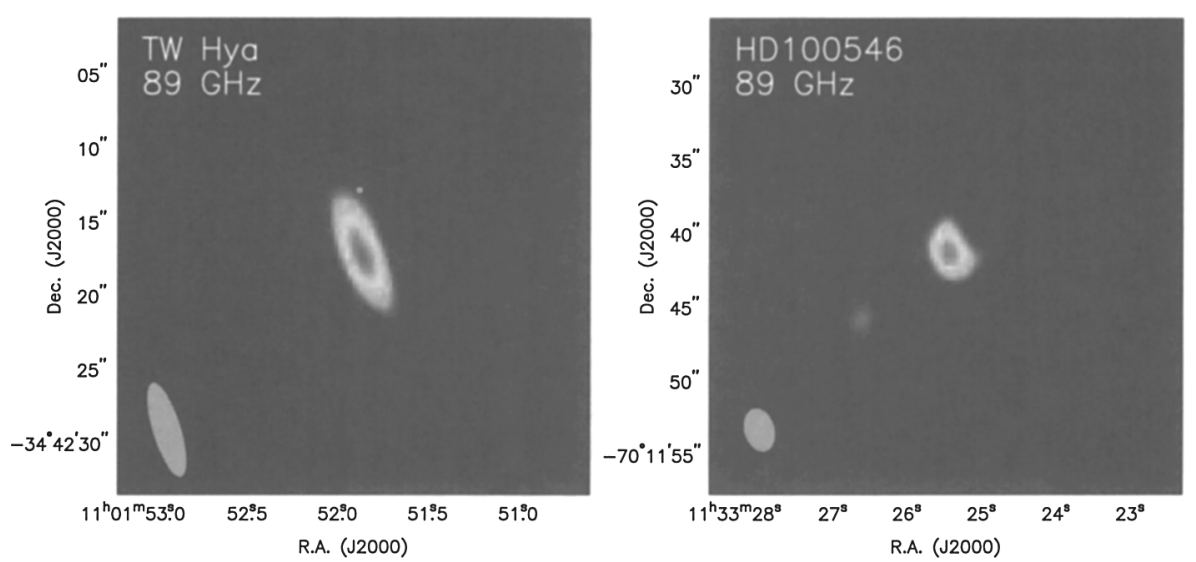

Figure 1. Images of $3.4 \mathrm{~mm}$ dust continuum emission from TW Hya and HD 100546 from the Australia Telescope Compact Array with $\sim 2^{\prime \prime}$ resolution and $\sim 3 \mathrm{mJy}$ rms sensitivity (Wilner et al. 2003).

\section{Imaging Dust Continuum Emission: Surveys}

Imaging dust continuum emission from the disks around nearby young stars at $\sim 1^{\prime \prime}$ resolution and $\sim$ mJy sensitivity, already sufficient to detect a small fraction of the minimum mass Solar Nebula, has become routine. The literature is filled with examples, and Figure 1 shows a recent result, the first $3 \mathrm{~mm}$ images of disks obtained with the first three upgraded antennas of the Australia Telescope Compact Array (Wilner et al. 2003). The dust emission from the disks around TW Hya and HD 100546 is well detected but just marginally resolved. Interferometric observations like these still require a significant investment of observing time, and at most a few objects may be imaged in a single Earth rotation synthesis track of $\sim 8$ hours duration.

With considerable effort, several interferometric imaging surveys of large samples of $\mathrm{T}$ Tauri stars now have been completed. The first major survey was performed with the IRAM PdBI at $2.7 \mathrm{~mm}$ (Dutrey et al. 1996), which resulted in the very important measurement of well-resolved sizes for a subset of the sample. Careful modeling of the observations implied disks around single stars with large outer radii, $R>150 \mathrm{AU}$, rather shallow radial density profiles, and significant molecular depletion. It is important to realize that detailed modeling has become an essential part of the analysis of high resolution millimeter data. A simple Gaussian fit may provide an indication that a disk is resolved, but a full-width-half-maximum "size" generally does not correspond to any relevant physical quantity, as realistic disks have brightness distributions that are far from Gaussian (Mundy et al. 1996). Following Beckwith et al. (1990), many observers have adopted simple power law parameterizations of disk surface density and temperature, i.e. $\Sigma \propto r^{-p}$ and $T \propto r^{-q}$, and this simplification has enabled considerable progress, in part because the fitted parameters may be readily related to plausible physical scenarios (Wilner \& Lay 2000).

A potential complication in the interpretation of the disk sizes inferred from observations at just one wavelength is that the disk models are potentially 


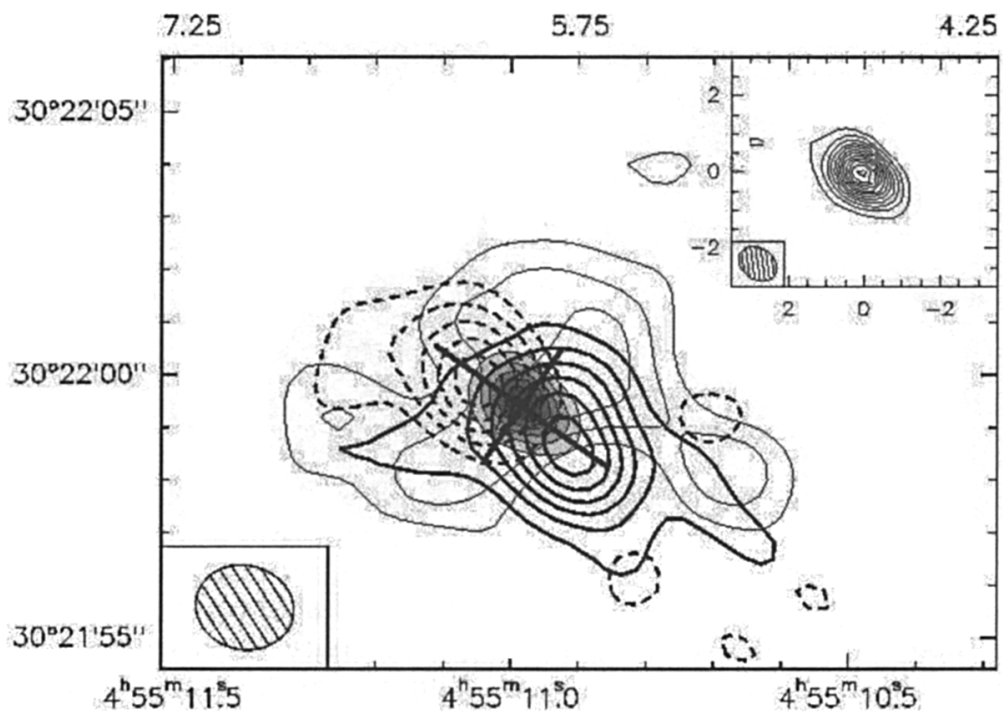

Figure 2. Images of GM Aur from the IRAM PdBI in $1.3 \mathrm{~mm}$ dust continuum emission (greyscale and insert) and $\mathrm{CO} \mathrm{J}=2-1$ emission (contours). The cross indicates the position, orientation, and aspect ratio of the $1.3 \mathrm{~mm}$ continuum peak, which shows excellent agreement with the disk rotation traced by the molecular line emission.

degenerate in the radial distributions of opacity and temperature. Guilloteau, Dutrey \& Gueth (1997) report that a "mini-survey" of T Tauri stars with the IRAM PdBI at $1.3 \mathrm{~mm}$ with the highest resolution available confirms the initial measurements of disk sizes derived from the earlier $2.7 \mathrm{~mm}$ survey.

Dust emission from disks is sometimes regarded as "smaller" in extent than molecular line emission from disks, but this is an illusion that is easily explained by opacity effects. Figure 2 shows high resolution images of $1.3 \mathrm{~mm}$ dust emission and $\mathrm{CO} \mathrm{J}=2-1$ line emission from GM Aur obtained with the IRAM PdBI (Dutrey et al. 1998). For a power law disk model, the optically thin dust brightness falls steeply, $T_{B} \propto r^{-(p+q)}$, while the CO line emission remains optically thick to large radii and reflects temperature, and the brightness falls less steeply, $T_{B} \propto r^{-q}$. The low brightness dust emission at large radii falls well below the sensitivity threshold, which accounts for the apparent size difference. Note also that observations of dust emission may be pushed to higher angular resolution than molecular line emission (with comparable signal-to-noise), because the dust emission is not Doppler limited in bandwidth by disk rotation. The inset in Figure 2 shows the dust emission at $0 . ' 6$ resolution, a synthesized beam area a factor of 3.5 smaller than that for the CO $\mathrm{J}=2-1$ line emission. And it is clear from the strength of the dust emission that the instrumental sensitivity is sufficient for substantially higher resolution imaging, if longer baselines were available, to probe the inner regions of the disk.

A second major survey that resolves the dust emission from a sample of $\mathrm{T}$ Tauri stars was recently completed using the NMA at $2 \mathrm{~mm}$ (Kitamura et al. 2002). Figure 3 shows images of 12 disk sources in Taurus from this survey, 

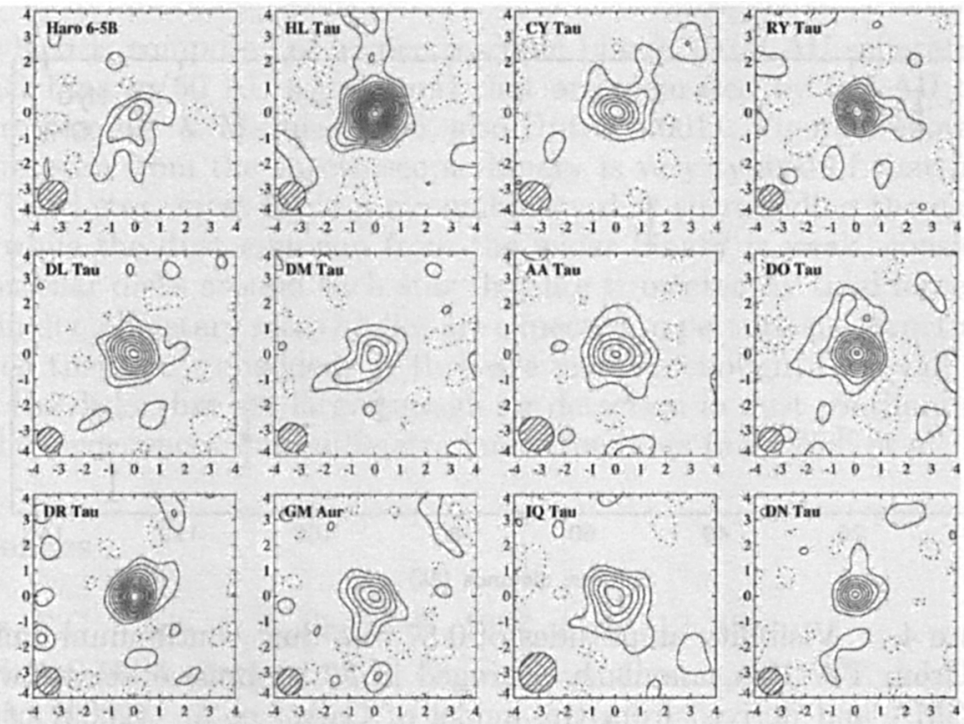

Figure 3. Images of $2 \mathrm{~mm}$ dust continuum emission from $\mathrm{T}$ Tauri stars obtained with the NMA at $\sim 1^{\prime \prime}$ resolution (Kitamura et al. 2002).

where the data were interpreted in the context of power law disk models and also viscous accretion disk models that lack a sharp outer edge. Again, relatively shallow surface density distributions are indicated, with values of the power law index $p$ in the range 0 to 1 , although the detailed correspondence of the derived values with previous determinations show considerable scatter. In any case, the uniform nature of this data set and analysis allowed for an investigation of correlations between the derived disk parameters and various evolutionary markers. An intriguing result is that the disk outer radius increases with decreasing $\mathrm{H} \alpha$ luminosity, perhaps due to radial expansion with age, as expected in accretion disks by transfer of angular momentum.

\section{Testing Disk Structure Models}

Theoretical models of disk structure and evolution are becoming more sophisticated, as more physics is incorporated and better computational methods are implemented. An important trend is to interpret high angular resolution millimetre data in the context of these physical models, instead of simple power law parameterizations. Several groups have developed self-consistent treatments of disks around young stars in radiative and hydrostatic equilibrium, employing radiative transfer schemes with various degrees of complexity (e.g. D'Alessio, Calvet \& Hartmann 2001, Dullemond \& Natta 2003). Figure 4 shows a comparison of recent SMA $0.87 \mathrm{~mm}$ dust continuum observations of the young star TW Hya (Qi et al., in preparation) with the predictions of the irradiated accretion disk model of Calvet et al. (2002), which matches the spectral energy distribution from optical to radio wavelengths and is consistent with resolved $7 \mathrm{~mm}$ VLA observations. Because the disk is viewed face-on and the radial brightness 


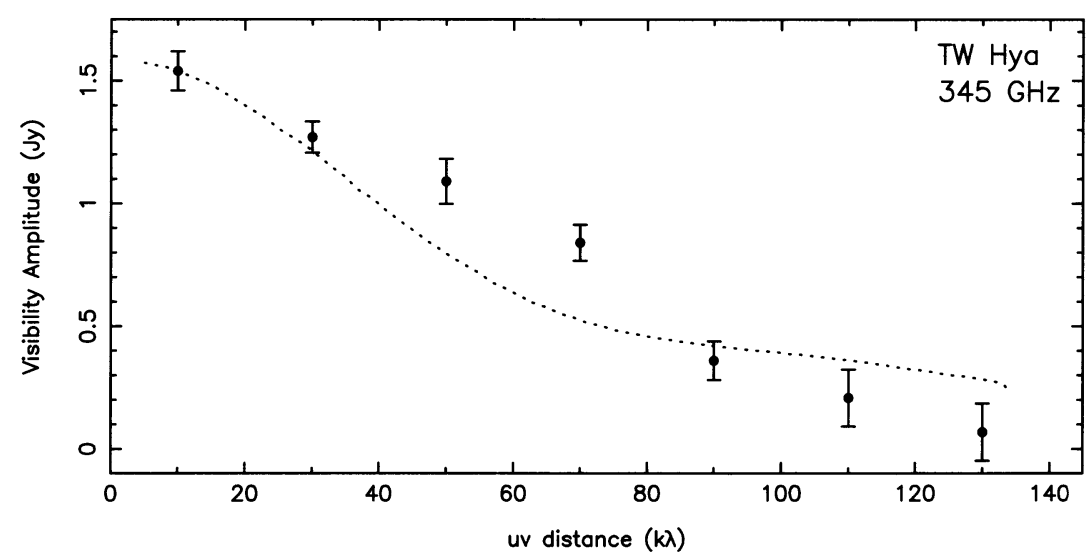

Figure 4. Visibility amplitudes of $0.87 \mathrm{~mm}$ dust continnuum emisssion from TW Hya, annularly averaged in $20 \mathrm{k} \lambda$ bins, observed with the SMA and derived from the model of Calvet et al. (2002) of an irradiated accretion disk (Qi et al. 2003). The error bars represent \pm 1 standard deviation.

distribution falls off steeply with radius, this comparison is best done via annular averaging in the visibility domain. The disk model, which was not developed to fit these data, provides an excellent match.

Simple considerations serve to illustrate the connections between accretion disk theory and the resulting surface density structure. For steady accretion, the surface density is given by $\Sigma=\dot{M} / 3 \pi \nu$ away from the boundaries, where $\nu=\alpha c_{s} H$ is the kinematic viscosity, parameterized by a local velocity (the sound speed, $c_{s}$ ), scale length (the scale height, $H$ ) and a dimensionless parameter $(\alpha<$ 1). The surface density may be expressed as $\Sigma \propto\left(r^{3 / 2} T_{m}\right)^{-1}$, where $T_{m}$ is the local midplane temperature whose value is determined by the balance of heating by stellar irradiation and viscous processes, and cooling through radiative losses. For a disk with heating dominated by stellar irradiation, the flaring of the outer parts tends to drive the temperature distribution to $T_{m} \propto r^{-1 / 2}$, except in the innermost regions where the disk becomes optically thick to its own radiation, and $\Sigma \sim r^{-1}$ (see Figure 5 of D'Alessio et al. 2001).

\section{Effects of Companions}

The preceding discussion concerns single stars, but most stars are known to be in multiple systems. The presence of stellar companions introduces considerable complexity in the structure and evolution of circumstellar disks. It has been known since the early single dish surveys that the millimeter continuum fluxes of pre-main-sequence binary systems are generally lower than single stars, presumably because the circumstellar disks in binary systems are subject to tidal truncation within the Roche lobes of the individual stars. An illustration is provided by high resolution $1.3 \mathrm{~mm}$ observations of the quadruple system UZ 
Tau, which is composed of a spectroscopic binary (0.03 AU separation) and a compact binary (50 AU separation) that are separated by 500 AU on the sky (Jensen, Koerner \& Mathieu 1996; also Dutrey 2001). Figure 5 shows that the dust emission from the spectroscopic binary is very typical of that from a single $\mathrm{T}$ Tauri star, most likely a circumbinary disk surrounding the close pair of stars, while the dust emission from the wider binary is weak, consistent with circumstellar disks around each star that are truncated by tidal forces.

Finally, planetary mass bodies are expected to perturb the structure of disks in which they are embedded. If they are massive enough, they will open gaps within the disks that are large enough for detection in dust continuum emission with the next generation millimetre interferometers (e.g. Wolf et al. 2002).

\section{References}

Adams, F.C., Lada, C.J., Shu, F.H. 1987, ApJ, 312, 788

Beckwith, S.V.W., Sargent, A.I., Chini, R., \& Gusten, R. 1990, AJ, 99, 924

Beckwith, S.V.W., Sargent, A.I. 1991, ApJ, 381, 250

Calvet, N., D'Alessio, P., Hartmann, L., Wilner, D., Walsh, A., Sitko, M. 2002, ApJ, 568, 1008

Chiang, E.I., Joung, M.K., Creech-Eakman, M.J., Qi, C., Kessler, J.E., Blake, G.A., van Dishoeck, E.F. 2001, ApJ, 547, 1077

Dullemond, C.P., Natta, A. 2003, A\&A, 408, 161

Dutrey, A., Guilloteau, S., Duvert, G. Prato, L., Simon, M., Schuster, K., Menard, F. 1996, A\&A, 309, 493

Dutrey, A., Guilloteau, S., Prato, L., Simon, M., Duvert, G., Schuster, K., Menard, F. 1998, A\&A, 338, L63

Dutrey, A. 2001, proceedings of IAU Symposium No. 200, eds. Hans Zinnecker and Robert D. Mathieu, p. 219.

Guilloteau, S., Dutrey, A., Gueth, F. 1997, proceedings of IAU Symposium No. 182, eds. Bo Reipurth and Claude Bertout, p. 365

Jensen, E.L.N., Koerner, D.W., Mathieu, R.D. 1996, AJ, 111, 2431

Kitamura, Y., Momose, M., Yokogawa, S., Kawabe, R., Tamura, M. Ida, S. 2002, ApJ, 581, 357

Mathieu, R.D., Ghez, A.M., Jensen, E.L.N., Simon, M., in Protostars and Planets IV, eds. Mannings, V., Boss, A.P., Russell, S.S., p. 703

Mundy, L.G., Looney, L.W., Erickson, W. Grossman, A., Welch, W.J., Forster, J.R., Wright, M.C.H. et al. 1996, ApJ, 464, L169

Wilner, D.J., Lay, O.P. 2000, in Protostars and Planets IV, eds. V. Mannings, A. Boss and S. Russell, p. 509

Wilner, D.J. 2002, in "The Origins of Stars and Planets: The VLT View", Springer-Verlag series of ESO Astrophysics Symposia, eds. J. Alves and M.J. McCaughrean, p. 311

Wilner, D.J., Bourke, T.L., Wright, C.M., Jorgensen, J.K., van Dishoeck, E.F., Wong, T. 2003, ApJ, 596, 597

Wolf, S., Gueth, F., Henning, T., Kley, W. 2002, ApJ, 566, L97 


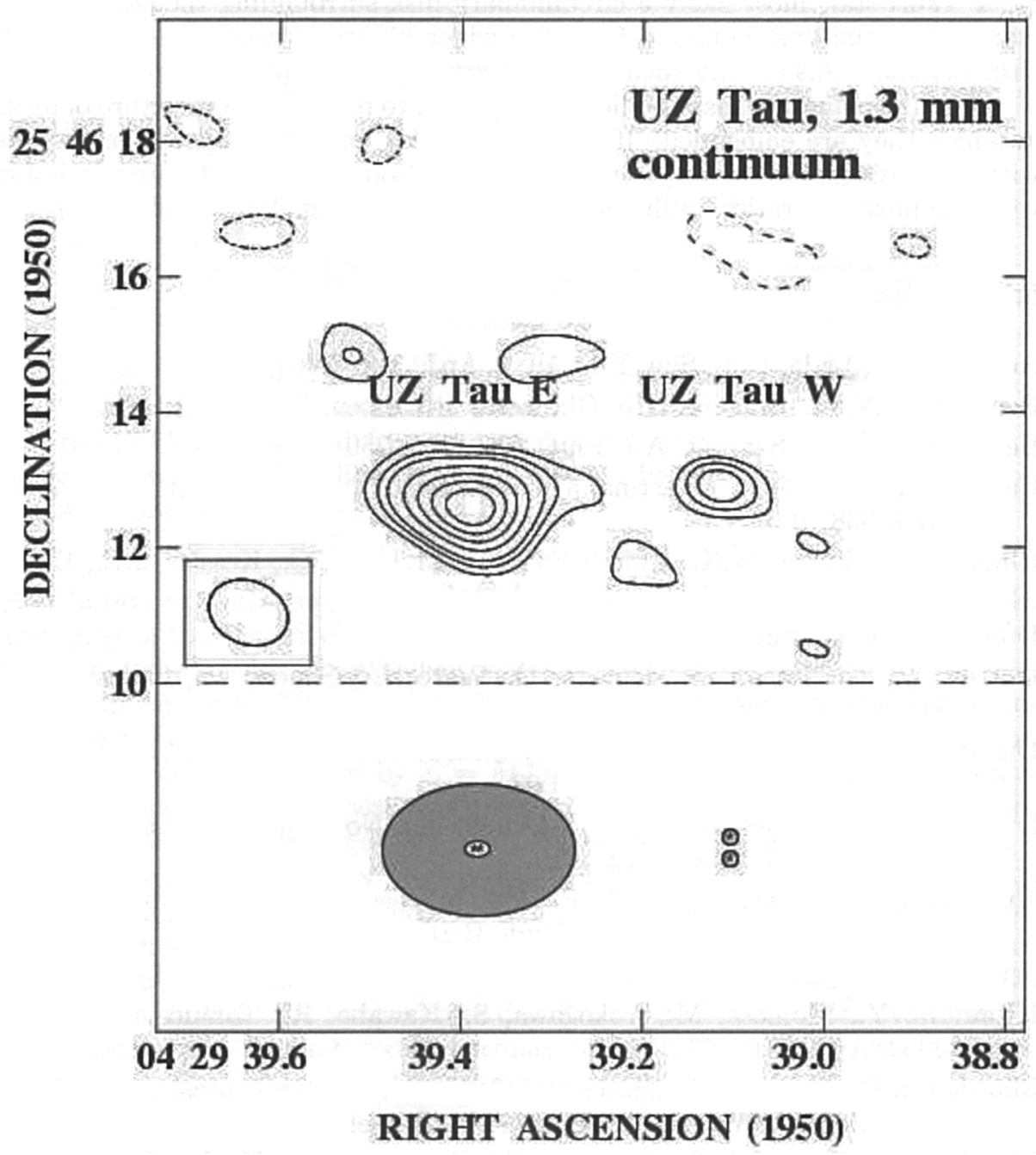

Figure 5. OVRO $1.3 \mathrm{~mm}$ dust continuum image of of UZ Tau and a schematic model of this quadruple system (Mathieu et al. 2000). The eastern spectroscopic binary shows strong emission a massive circumbinary disk, while the western wider binary shows much less emission, likely because the circumstellar disks are tidally truncated. 